\title{
A 3D co-culture of three human cell lines to model the inflamed intestinal mucosa for safety testing of nanomaterials
}

\author{
Julia Susewind ${ }^{1}$, Cristiane de Souza Carvalho-Wodarz ${ }^{1}$, Urska Repnik ${ }^{3}$, Eva-Maria Collnot ${ }^{1,2}$, \\ Nicole Schneider-Daum $^{1}$, Gareth Wyn Griffiths ${ }^{3}$, and Claus-Michael Lehr ${ }^{1,2}$ \\ ${ }^{1}$ Department of Drug Delivery, Helmholtz Institute for Pharmaceutical Research Saarland (HIPS), Helmholtz Centre for Infection Research (HZI), \\ Saarbrücken, Germany, ${ }^{2}$ Department of Pharmacy, Biopharmacy and Pharmaceutical Technology, Saarland University, Saarbrücken, Germany, and \\ ${ }^{3}$ Department of Biosciences, University of Oslo, Blindernveien, Oslo, Norway
}

\section{Abstract}

Oral exposure to nanomaterials is a current concern, asking for innovative biological test systems to assess their safety, especially also in conditions of inflammatory disorders. Aim of this study was to develop a 3D intestinal model, consisting of Caco-2 cells and two human immune cell lines, suitable to assess nanomaterial toxicity, in either healthy or diseased conditions. Human macrophages (THP-1) and human dendritic cells (MUTZ-3) were embedded in a collagen scaffold and seeded on the apical side of transwell inserts. Caco-2 cells were seeded on top of this layer, forming a 3D model of the intestinal mucosa. Toxicity of engineered nanoparticles (NM101 $\mathrm{TiO}_{2}, \mathrm{NM} 300 \mathrm{Ag}, \mathrm{Au}$ ) was evaluated in non-inflamed and inflamed co-cultures, and also compared to non-inflamed Caco-2 monocultures. Inflammation was elicited by IL-1 $\beta$, and interactions with engineered NPs were addressed by different endpoints. The 3D co-culture showed well preserved ultrastructure and significant barrier properties. Ag NPs were found to be more toxic than $\mathrm{TiO}_{2}$ or Au NPs. But once inflamed with IL-1 $\beta$, the co-cultures released higher amounts of IL-8 compared to Caco-2 monocultures. However, the cytotoxicity of Ag NPs was higher in Caco-2 monocultures than in 3D co-cultures. The naturally higher IL-8 production in the co-cultures was enhanced even further by the Ag NPs. This study shows that it is possible to mimic inflamed conditions in a 3D co-culture model of the intestinal mucosa. The fact that it is based on three easily available human cell lines makes this model valuable to study the safety of nanomaterials in the context of inflammation.
\end{abstract}

Keywords

Caco-2, cytotoxicity, intestine, nanoparticles

\section{History}

Received 16 September 2014

Revised 6 January 2015

Accepted 6 January 2015

Published online 4 March 2015

\section{Introduction}

Engineered nanomaterials (ENs) are usually employed in food packaging or as additives in various food products, thereby increasing the chance of unintended human exposure. Increased exposure to particulate matter has been one of the main environmental factors associated with autoimmune and inflammatory diseases, especially in the intestine (Alexis et al., 2014; Kish et al., 2013). Studies show that exposure to microparticles has been linked to the occurrence of inflammatory bowel diseases (IBD), such as Crohn's disease (Powell et al., 2010). Food often contains many non-nutrient microparticles, like soil and dust or food additives, which can be taken up by mucosal cells, resulting in inflammatory reactions (Mahmud \& Weir, 2001). Nanoparticles (NPs) can be inhaled, e.g. carbon NPs from laser printers (Theegarten et al., 2010), but also swallowed, the latter case being reported to cause diarrhea and histological alterations

Correspondence: Prof. Dr. Claus-Michael Lehr, Department of Drug Delivery, Helmholtz-Institute for Pharmaceutical Research, Helmholtz Centre for Infection Research (HZI), Campus A 4.1, 66123 Saarbrücken, Germany. Tel: +49 (0)681 302-2964. Fax: +49 (0)681 302-4677. E-mail: lehr@mx.uni-saarland.de typical of Crohn's disease (Gaiser et al., 2009, 2012). Thus, there is an urgent need to better understand the interaction between nanomaterials and the intestinal mucosa, especially in the context of local inflammatory events and diseases (Lefebvre et al., 2014).

Engineered nanomaterials are also used in innovative drug delivery systems as they can target drugs to their site of action and can protect the cargo from degradation (Beloqui et al., 2014). Therefore, it is essential to thoroughly characterize the safety of ENs for humans. However, the complexity of biological systems makes it difficult to precisely assess the impact of nanomaterials. This holds true in particular for animal models, which is due to their complexity are essentially "black boxes", which are difficult to handle and also pose ethical challenges. Therefore, there is a need for advanced cell and tissue based in vitro models that allow studying the effects of NPs under well-controlled conditions by monitoring endpoints of clinical relevance. Currently, the enterocyte cell line Caco-2 is a well-established model for the human intestinal barrier. A number of in vitro studies on the toxicity of ENs have used the Caco-2 cell line to assess cytotoxicity in the gastrointestinal tract (GI) and systemic uptake after oral exposure (Fisichella et al., 2012; Koeneman et al., 2010). However, the complex physiology of the intestinal mucosa is insufficiently modeled with only one cell type, which might explain the limited correlation between in vivo and in vitro studies (Foger et al., 2008; Frick et al., 1998; Walter et al., 1996). 
One obvious shortcoming of the Caco-2 monoculture model is the lack of immune-competent cells, which are clearly important in the context of inflammatory disorders, such as IBD. Interactions between nanomaterials and immune cells can lead to inflammatory reactions with pro-inflammatory cytokine release (Dobrovolskaia \& McNeil, 2007) and cytotoxic effects (Bisping et al., 2001). Nanoparticle uptake by immune cells is an important issue related to nanotoxicity (Dobrovolskaia et al., 2008). Several NP properties, such as the physicochemical properties of size and charge, might influence cellular particle uptake. Studies show that particles with cationic or anionic surface charges are more attractive to phagocytes than neutral particles (Ayala et al., 2013) and it is known that particles below $200 \mathrm{~nm}$ in diameter show poor uptake by macrophages in comparison to particles between 0.5 and $3 \mu \mathrm{m}$ (Desjardins \& Griffiths, 2003). Several groups have used in vitro cell models to evaluate NP toxicity either in monocultures (Fisichella et al., 2012; Gaiser et al., 2012, 2013; Koeneman et al., 2010) or in co-cultures (Muller et al., 2010; RothenRutishauser et al., 2008). Indeed, in vitro models containing two or more types of cells, able to interact with each other and with nanomaterials, seem more able to capture the complexity of the in vivo system. Our research group has previously established a 3D in vitro model of the intestinal mucosa consisting of the Caco-2 intestinal epithelial cell line with primary blood derived dendritic cells and macrophages embedded in a collagen type I gel (Leonard et al., 2010). This model shows relevant barrier properties for studying NP translocation. It appears to reflect typical pathophysiological changes that are observed in the state of inflammation in vivo, such as decreased epithelial barrier function, increased production of pro-inflammatory cytokines and an alleviation of such symptoms when anti-inflammatory formulations for the treatment of IBD were tested (Leonard et al., 2012). However, as it is based on primary derived immune cells, this model suffered from a relatively high variability, which makes it more difficult to standardize, and limits its capacity for use in high-throughput screenings.

The aim of the present study was to improve this in vitro model by replacing the primary macrophages and dendritic cells by cell lines, THP-1 and MUTZ-3, respectively, in order to have a valuable and reproducible model to assess cytotoxicity of engineered NPs. For this purpose, silver (Ag), titanium dioxide $\left(\mathrm{TiO}_{2}\right)$ and gold $(\mathrm{Au}) \mathrm{NPs}$ were used. These particles were chosen based on their relevance for oral exposure as well as for their use in other toxicological studies: Ag nanoparticles are frequently used in commercial products because of their antimicrobial properties. A number of studies have described Ag nanoparticles as being able to induce toxicity in different species and uptake from the intestine has been reported in animal models (Beer et al., 2012; Loeschner et al., 2011). $\mathrm{TiO}_{2}$ nanoparticles are present in many foods, especially sweets like candies and marshmallows (Weir, 2012) and as whitener in toothpaste; Au NPs may also be included in packaging (Frohlich \& Roblegg, 2012), but nanomedicines/diagnostics and nanoelectronics are more likely routes of exposure. The toxic potential and uptake pathway of Au NPs are still unclear (Mironava et al., 2010).

\section{Methods}

\section{Cell cultures}

Caco-2, THP-1 and MUTZ-3 cells were passaged and cultured as described in SI.

\section{Three-dimensional triple cell culture}

Differentiated THP-1 and MUTZ-3 cells were embedded in a $80 \%(w / v)$ solution of type I bovine collagen (Advanced Biomatrix,
Tucson, AZ) pH 7.4 with human AB serum (Invitrogen, Madison, WI) and RPMI; $150 \mu \mathrm{l}$ of this solution was pipetted on top of transwell filter inserts (Corning Incorporated, Acton, MA, $0.4 \mu \mathrm{m}$ pore size, $1.12 \mathrm{~cm}^{2}$ area) resulting in a seeding density of $10^{4}$ cells of each cell type per well. After 1-hour incubation at $37^{\circ} \mathrm{C}$ and $5 \%$ $\mathrm{CO}_{2}, 6 \times 10^{4}$ Caco- 2 cells were seeded on top of the formed collagen gel layer. The co-culture was incubated with $500 \mu \mathrm{l}$ DMEM/1\% FBS/1\% NEAA with 1\% Pen/Strep (PAA, Pasching, Austria) in the apical compartment and $1.5 \mathrm{ml}$ RPMI 1640/10\% FBS with $1 \%$ Pen/Strep in the basolateral compartment. Medium was changed every second day. The co-culture cells were grown for 21 days. To induce inflammation, $10 \mathrm{ng} / \mathrm{ml} \mathrm{IL-1} \beta$ was added to the apical compartment and incubated for 2 days. After this time, the medium containing IL-1 $\beta$ was removed and NPs were incubated for $24 \mathrm{~h}$. Non-inflamed co-cultures were run in parallel and also incubated with NPs to evaluate the interaction with healthy cells.

\section{Transmission electron microscopy}

Samples were prepared as described in SI.

\section{Immunofluorescence staining}

Human macrophages or dendritic cells were pre-stained with $2 \mu \mathrm{g} / \mathrm{ml}$ of 6-carboxyfluorescein diacetate before they were used in the co-culture. The 3D co-culture was fixed with $3 \%$ paraformaldehyde. Tight junctions were stained with monoclonal mouse anti-occludin (Zymed, San Francisco, CA; 1:200) and Alexa633 anti-mouse (Life Technologies, Darmstadt, Germany; 1:500), nuclei were stained with DAPI (Life Technologies, Darmstadt, Germany). Detailed protocol is described in SI.

\section{Nanoparticle preparation and characterization}

NM-300 Ag nanoparticles, PVP-capped (<20 nm) and NM-101 $\mathrm{TiO}_{2}$ nanoparticles $(7-10 \mathrm{~nm})$ were purchased from JRC (Joint Research Centre), Ispra, Italy. Au phosphine-capped nanoparticles $(15$ and $80 \mathrm{~nm})$ were kindly provided by Dr W. Kreyling, Institute of Lung Biology and Disease, Helmholtz Zentrum, Munich.

Particles were dispersed in DMEM/10\% fetal bovine serum (FBS)/1\% non-essential amino acids (NEAA). All nanoparticles were prepared and characterized as described by Ucciferri et al. (2014). NP concentrations tested in cell models are shown in Table S1.

All nanoparticle solutions were free of endotoxins.

\section{Cell viability}

In order to carry out lactate dehydrogenase (LDH) and Alamar Blue assays, Caco-2 monocultures were seeded in 96-well plates at $2 \times 10^{4}$ cells/well and grown for 7 days. Cells were incubated with different concentrations of NPs for $24 \mathrm{~h}$ at $37^{\circ} \mathrm{C}$ and $5 \%$ $\mathrm{CO}_{2}$. Afterwards the supernatant was collected to measure $\mathrm{LDH}$ production, and adherent cells were used for Alamar Blue assays. Cell supernatants were incubated with $\mathrm{LDH}$ reagent (LDH detection kit, Roche, Mannheim, Germany) in the dark for $3 \mathrm{~min}$ at RT and excitation was measured at $492 \mathrm{~nm}$. Adherent cells were incubated with Alamar Blue reagent (CellTiter-Blue, Roche, Mannheim, Germany) for 30 and $150 \mathrm{~min}$, and the formation of violet Resofurin salt was measured at $590 \mathrm{~nm}$ using a plate reader (TECAN, Männerdorf, Switzerland). The specific metabolic activity was calculated as: $\left(\mathrm{Abs}_{2 \mathrm{~h} 30^{\prime}}-\mathrm{Abs}_{30^{\prime}}\right) /$ $120^{\prime}$. Sample data were normalized to the non-treated medium control.

The viability of the triple cultures incubated with different concentrations of NPs was also evaluated with LDH and Alamar Blue assays in a similar manner. 


\section{Transepithelial electrical resistance}

Transepithelial electrical resistance (TEER) of mono- and triple cultures was measured as described by Leonard et al. (2010). Only cell cultures representing TEER values between 400 and $600 \Omega * \mathrm{~cm}^{2}$ were used for experiments.

\section{IL-8 cytokine measurement}

Monoculture, inflamed and non-inflamed triple cultures were incubated with the different NPs for $24 \mathrm{~h}$ and IL-8 release was measured in cell culture supernatant with CBA Flex Set for IL-8 (BD Biosciences, Heidelberg, Germany) using a fluorescence activated cell sorter (FACS Calibur, BD Biosciences, Heidelberg, Germany). Analysis was done with FCAP array v1.01 cytometric bead array analysis software (BD Biosciences, Heidelberg, Germany).

\section{Quantitative RT-PCR}

Relative changes of mRNA transcription of the pro-inflammatory marker IL-8 after incubation with different nanoparticles were determined using qualitative real-time polymerase chain reaction (qRT-PCR). Cells were incubated with NPs for $24 \mathrm{~h}$, collected and lysed. Total RNA was extracted from the cells with QIAshredder (Qiagen, Hilden, Germany) and RNeasy Mini Kit (Qiagen, Hilden, Germany) according to the manufacturer's protocol. RNA amount was measured using a photometer (Eppendorf). A $500 \mu \mathrm{g}$ amount of RNA was transcribed into cDNA with a QuantiTect Transcription Kit (Qiagen, Hilden, Germany).

Quantitative PCR was conducted using QuantiTect Probe PCR Kit (Qiagen, Germany) according to the manufacturer's instruction. Three replicates were measured for every sample. Thirty-five cycles were measured $\left(10 \mathrm{~s}\right.$ : $94^{\circ} \mathrm{C}, 30 \mathrm{~s}$ : $\left.60^{\circ} \mathrm{C}\right)$. $\beta$-actin was used as a housekeeping gene. Sequences of primers and probes are shown in Table S2.

\section{Statistical analysis}

Data are presented as mean \pm SD. One-way ANOVA, Holm Sidak test, was used to compare results from different treatments at different time points. The ANOVA analysis was done with SigmaPlot 12.5. Significance was assumed at $* p<0.05$ or $*^{*} p<0.001$. Individual experiments were performed in triplicate and each experiment was performed twice. EC50 values were calculated with SigmaPlot 12.5 (Systat Software GmbH, Erkrath, Germany).

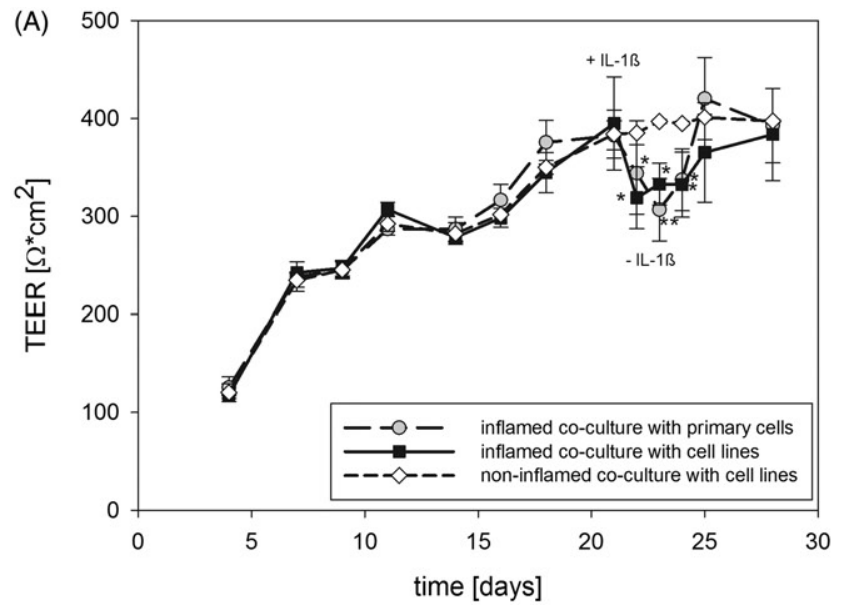

\section{Results}

\section{Replacement of primary cells by cell lines}

The characteristics of the 3D co-culture consisting of human cell lines were evaluated and compared to the already established 3D co-culture containing primary cells (Leonard et al., 2010). Therefore, 3D co-cultures containing primary immune cells were carried out in parallel to the 3D co-cultures with cell lines.

Epithelial barrier function was evaluated by measuring TEER. After 21 days, the Caco- 2 cells in the 3D co-cultures (both using cell lines and primary cells) reached TEER values between 350 and $450 \Omega * \mathrm{~cm}^{2}$, which characterizes a confluent monolayer (Figure 1A). At this time, the co-cultures were inflamed with $10 \mathrm{ng} / \mathrm{ml}$ of IL-1 $\beta$ added into the apical compartment. TEER values decreased significantly compared to a non-inflamed cell line-based co-culture, which agrees with previous models based on primary cells (Fang et al., 2010; Leonard et al., 2010). To evaluate the reversible effect of inflammation on barrier properties, IL-1 $\beta$ was removed from the co-cultures on day 23 . In the absence of IL-1 $\beta$, barrier properties of both cell line and primary cell based co-cultures recovered as observed in relation to TEER measurement (Figure 1A).

As an indication of inflammatory responses, IL-8 release from co-cultures and Caco-2 cell monoculture, was measured after IL-1 $\beta$ stimulation (Figure 1B). Co-culture containing either primary cells or cell lines showed progressive increase in the amount of IL-8 release during the investigated time period, reaching approximately $200 \mathrm{pg} / \mathrm{ml}$ after $48 \mathrm{~h}$. However, in Caco-2 monoculture only a slight IL-8 release could be detected even $48 \mathrm{~h}$ after stimulation. Additionally, Figure S1 shows IL-8 release of THP-1 and MUTZ-3 cells, which were seeded separately in the collagen layer under same conditions as the co-culture. Both cell types are able to produce IL- 8 after inflammation with IL-1 $\beta$.

\section{Morphological characterization of triple co-cultures}

The 3D co-culture consisting of three cell lines was evaluated by confocal microscopy (Figure 2) and transmission electron microscopy (Figure 3). Using confocal microscopy, some macrophages (green) and dendritic cells (green) could be observed situated between the epithelial cells, either in healthy or inflamed culture condition (Figure 2). Moreover, the tight junctions stained with an occludin antibody (red) showed a preserved structure in

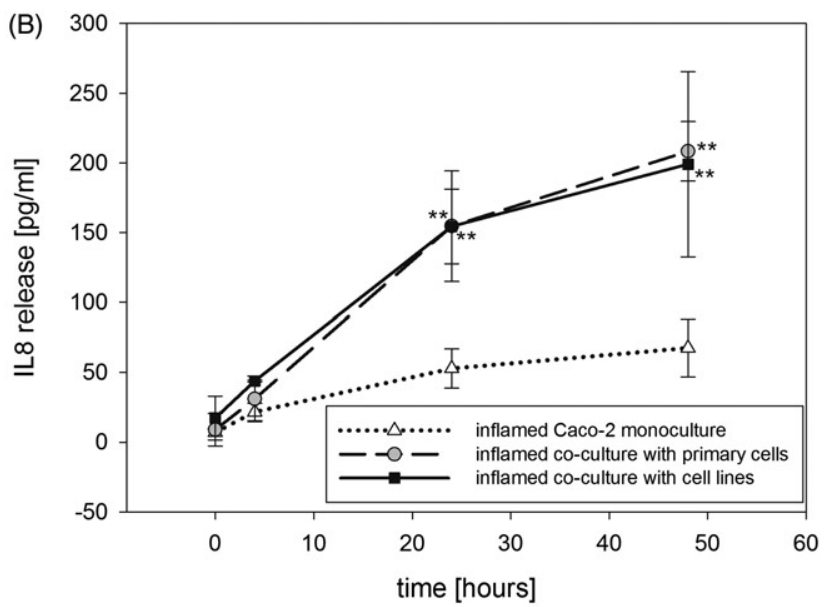

Figure 1. TEER (A) and IL-8 release (B) of co-culture setups after inflammation with IL-1 $\beta$. Co-cultures were grown for three weeks and inflamed at day 21 (arrow down). At day 23, IL-1 $\beta$ was removed (arrow up). Results of both inflamed co-cultures were compared to TEER of a non-inflamed coculture with cell lines. TEER decreases after treatment with IL-1 $\beta$ after $24 \mathrm{~h}$. After removal of IL-1 $\beta$ TEER values recover again after three to four days (A). IL-8 release was measured in co-cultures with cell lines and primary cells $0,4,24$ and $48 \mathrm{~h}$ after inflammation with IL-1 $\beta$ (B). Results were compared to IL-8 release from inflamed Caco- 2 monoculture (mean $\pm \mathrm{SD}, n=6$ from two independent experiments, ${ }^{*} p<0.05$, $* * p<0.001$ ). 


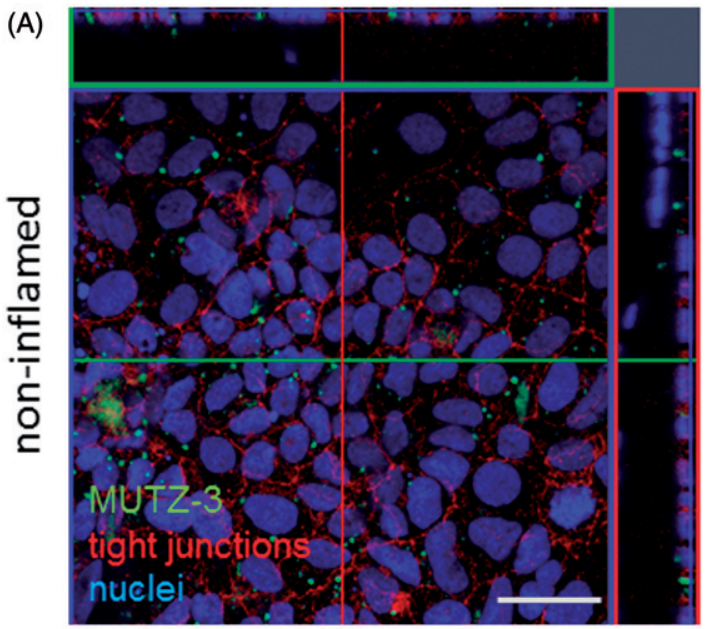

(B)
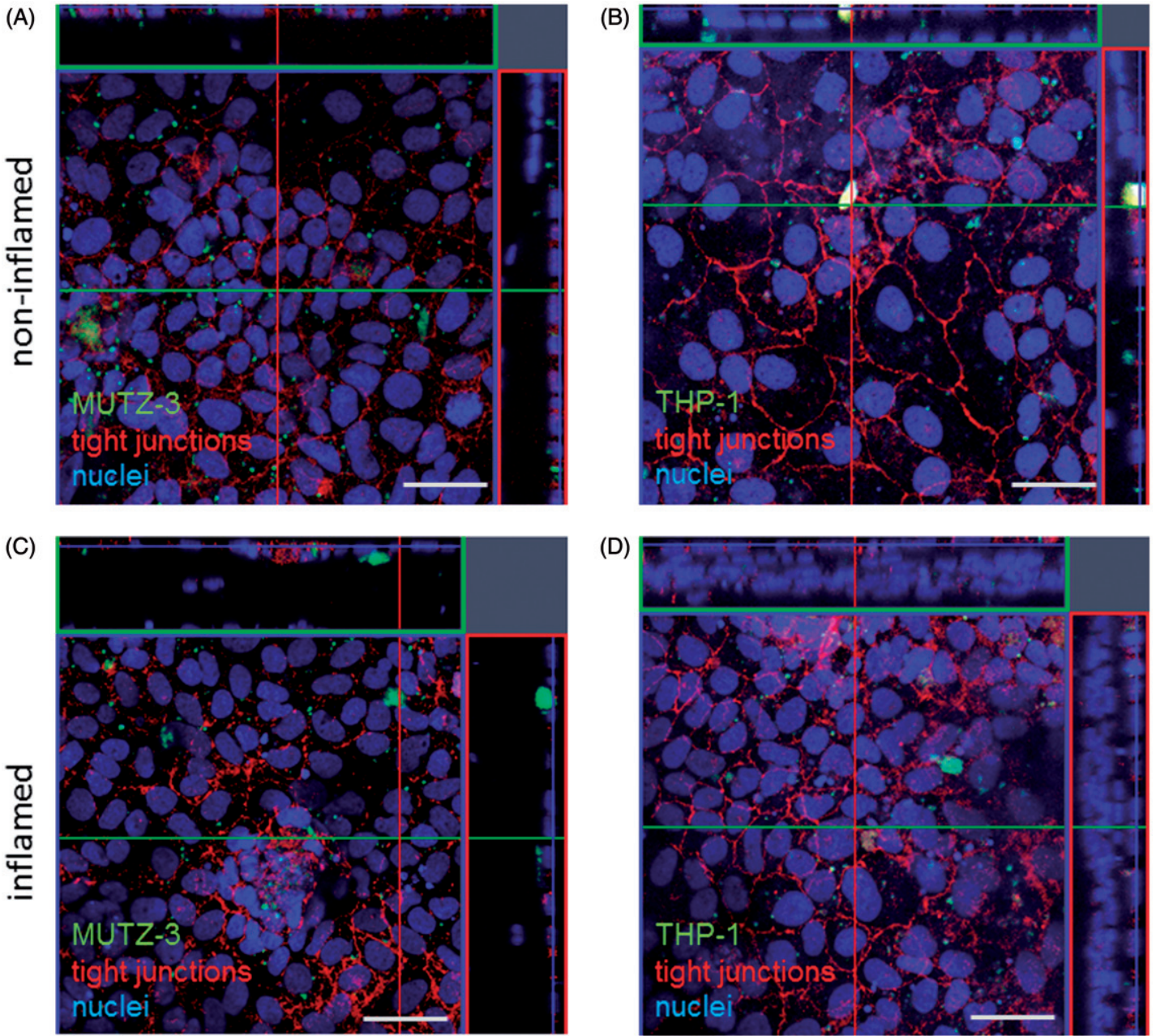

(D)

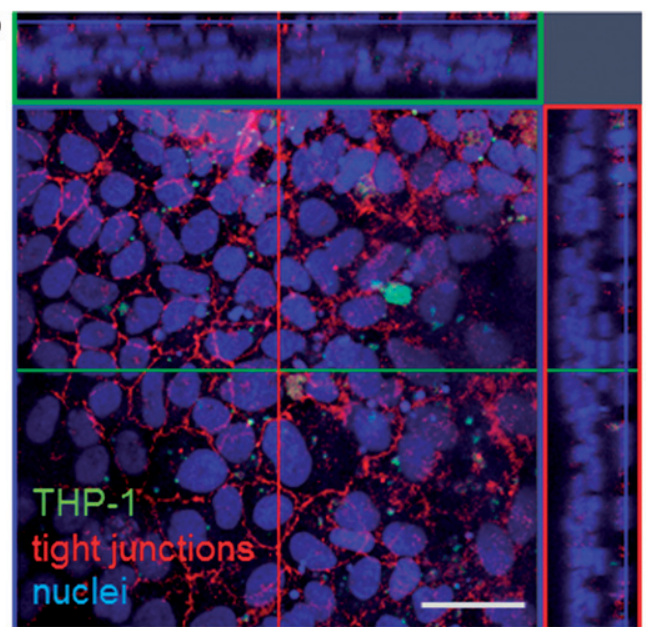

Figure 2. Confocal microscopy of inflamed triple culture. Z-Stacks of non-inflamed (A,B) and inflamed (C,D) co-culture; epithelial tight junctions are stained with an anti-occludin antibody; MUTZ-3 cells (A,C) or THP-1 cells (B,D) are pre-stained with CFDA-SE, cell nuclei are stained with DAPI. Tight junctions in the inflamed co-culture are disrupted and not as well defined as in the non-inflamed one. Macrophages and dendritic cells are in between or on top of the Caco-2 cells in both conditions. Scale bar: $50 \mu \mathrm{m}$.

healthy conditions, whereas in the inflamed co-culture the structure of the tight junctions was disrupted.

At the ultrastructural level, the 3D non-inflamed co-culture showed macrophages and dendritic cells embedded into the collagen layer and epithelial cells on top (Figure 3A). Macrophages and dendritic cells were also observed separately (Figure S2). Figure S2 shows that both cell types can be distinguished because of their amount of condensed heterochromatin in the nucleus. Dendritic cells show more condensed heterochromatin than macrophages, which can also be observed in Figure 3(A). Some macrophages were observed on top of Caco-2 cells, which provide evidence for the translocation of these immune cells through the epithelial barrier, in agreement with the confocal microscopy images. Immune cells could also be wandered through the Caco-2 cells before the formation of a tight monolayer. The epithelial cells formed a monolayer with characteristic microvilli (Figure 3B), tight junctions and desmosomes (Figure 3C and D). Furthermore, immune cells in the collagen layer were well preserved (Figure 3E) and made cell contacts (Figure 3F-i and ii). A similar ultrastructural pattern was observed in the inflamed co-culture system (data not shown).

\section{Nanoparticle characterization}

Engineered NPs were evaluated according to their dispersion behavior. The two Au NP batches could be easily dispersed in the culture medium at the tested concentrations. The measured hydrodynamic diameter was slightly larger than the nominal diameter according to the manufacturer (Table 1) with a polydispersity index (PDI) $<0.1$ even at high concentrations of $0.1 \mathrm{mg} / \mathrm{ml}$. The particles did not agglomerate in the cell culture medium, but a thin corona was formed likely from the adsorption of proteins or other components from the Caco-2 medium.

For $\mathrm{TiO}_{2}$ particles, the dispersibility was very poor in MilliQ water, but could be improved in cell culture medium due to the high FBS/protein concentration and steric stabilization of the particle dispersion. Nevertheless, agglomerates between 700 and $1200 \mathrm{~nm}$ were still formed with a high PDI (>0.4).

Ag NPs could not be evaluated using dynamic light scattering due to the interference with the laser in the zetasizer (Zetasizer Nano, Malvern Instruments, Herrenberg, Germany). Instead size and stability of the particle dispersion were determined using single particle tracking analysis (NanoSight, Malvern Instruments, Herrenberg, Germany), which employs a different laser wavelength. Mean hydrodynamic diameter of the particles was $120 \mathrm{~nm}$ hinting at particle cluster formation together with serum proteins. Size distribution was monomodal, no agglomeration was observed within $24 \mathrm{~h}$ of storage in cell culture medium at $4{ }^{\circ} \mathrm{C}$.

\section{Nanoparticle interaction with mono- and co-cultures}

The extent to which each cell type in the cell line-based co-culture could internalize NPs was investigated using transmission electron microscopy. It was observed that only Caco- 2 cells were able 


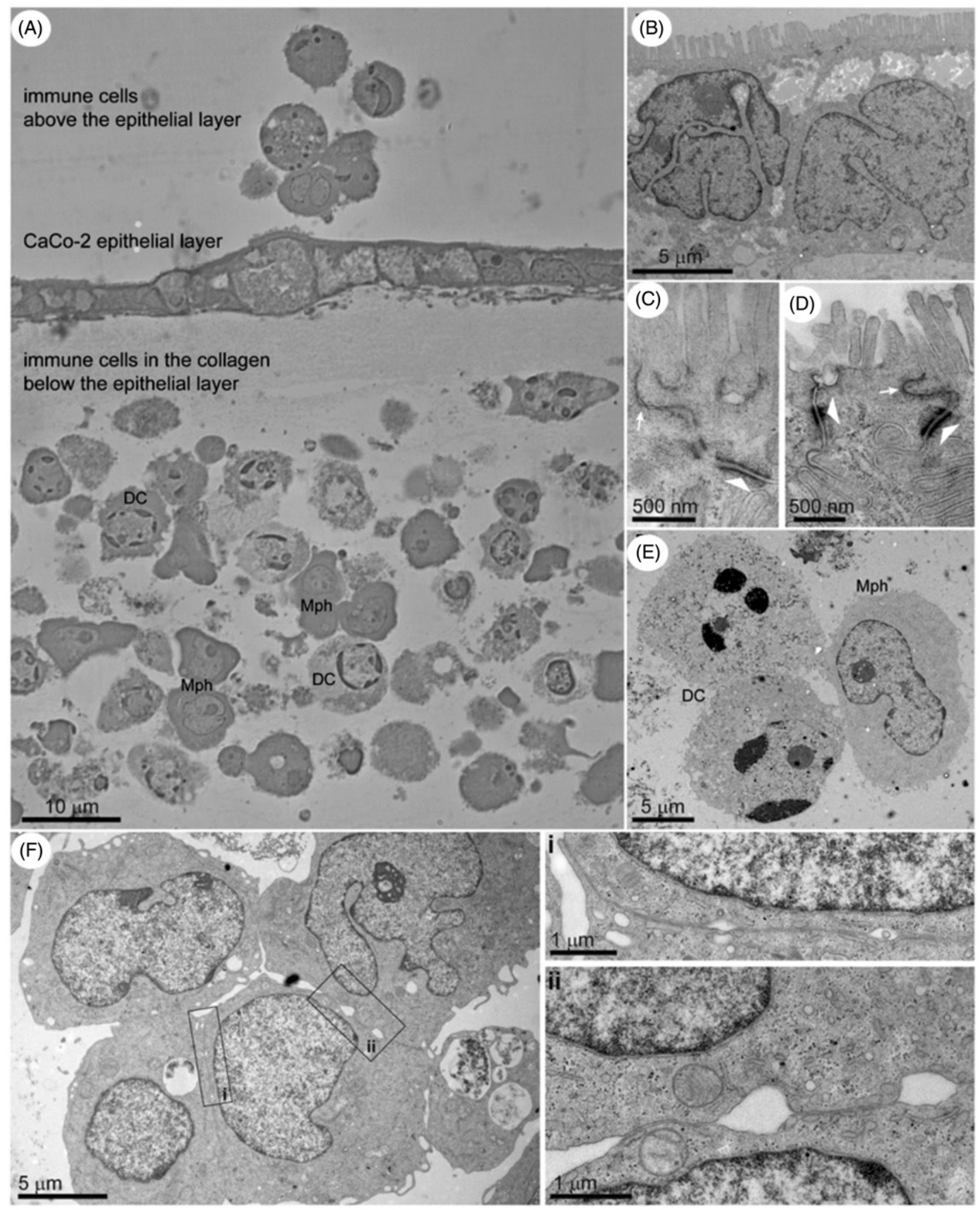

Figure 3. An overview of the non-inflamed triple culture. Caco-2 cells in the epithelial layer (B, C, D) with immune cells above and immune cells in the collagen gel below it (A). Cell junctions in the epithelial Caco-2 layer (tight junctions, slim arrows; desmosomes, block arrows) (C, D). Immune cells are represented by macrophages (Mph) and dendritic cells (DC) (E, F). Inserts (i, ii) show the interaction between immune cells in the collagen layer. (A): $2 \mu \mathrm{m}$ thick epon section analyzed with a wide field light microscope; (B-F): $70 \mathrm{~nm}$ thin epon sections analyzed with a transmission electron microscope.

Table 1. Dispersion behavior of ENs in DMEM cell culture medium at $0.1 \mathrm{mg} / \mathrm{ml}$ after $1 \mathrm{~h}$ of incubation.

\begin{tabular}{lcc}
\hline NPs & $\begin{array}{c}\text { Mean hydrodynamic } \\
\text { diameter [nm] }\end{array}$ & PDI \\
\hline $\mathrm{Au} 15$ & $51 \pm 6$ & $0.04 \pm 0.01$ \\
$\mathrm{Au} 80$ & $116 \pm 5$ & $0.04 \pm 0.02$ \\
$\mathrm{TiO}_{2}$ & $896 \pm 133$ & $0.42 \pm 0.14$ \\
$\mathrm{Ag}$ & $120 \pm 4$ & $\mathrm{NA}$ \\
\hline
\end{tabular}

to internalize $\mathrm{Au} \mathrm{NPs,} \mathrm{in} \mathrm{healthy} \mathrm{or} \mathrm{inflamed} \mathrm{conditions}$ (Figure S3).

To assess cytotoxicity, each of the NPs was incubated with both Caco-2 mono- and co-cultures in both inflamed and noninflamed conditions, according to the same protocol. Incubation with the highest employed concentration of Au NP types did not affect cell viability and there was no difference between the results of the Caco-2 mono- and co-culture setups, regardless of the state of inflammation. In contrast, the highest employed concentration of Ag NPs induced significant toxicity after $24 \mathrm{~h}$. The Caco-2 monoculture was more sensitive to Ag NPs than the co-culture model, while at this highest particle concentration there 

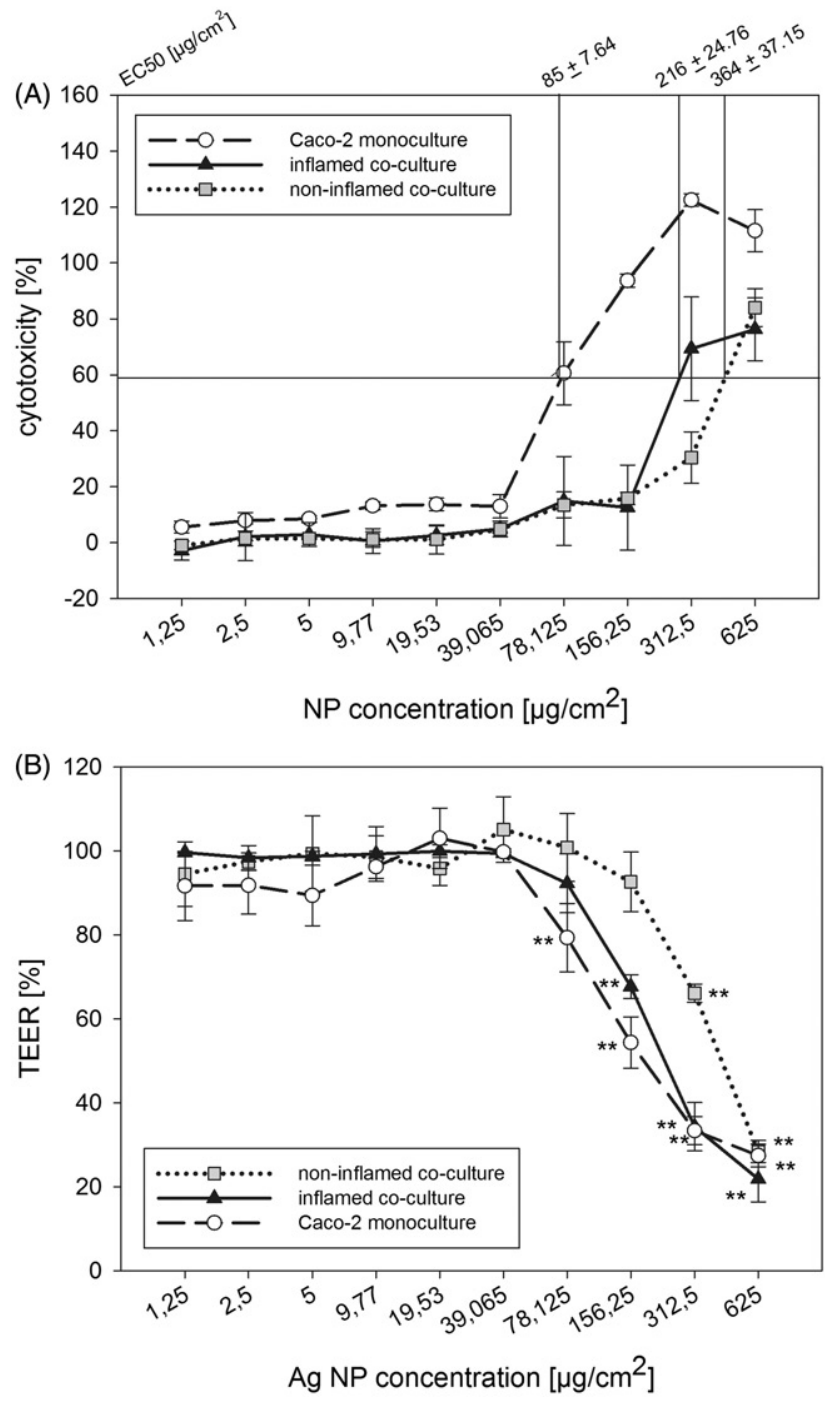

Figure 4. NP cytotoxicity after incubation with $\mathrm{Ag}$ for $24 \mathrm{~h}$. LDH release (A) and TEER (B) were measured for all Ag NP concentrations in non-inflamed Caco-2, inflamed and non-inflamed co-culture. TEER values after incubation with NPs were calculated as percentage of the TEER values before incubation (mean $\pm \mathrm{SD}, n=6$ from two independent experiments, ${ }^{*} p<0.001$ in comparison to TEER values of a non-treated control)

was no significant influence of the state of co-culture inflammation (Figure S4).

To be sure that the different cultivation conditions of Caco-2 monocultures and co-cultures did not influence the results, it was additionally shown that LDH and Alamar Blue assays show the same results, no matter if Caco-2 cells were grown in 96-well plates or on transwell filter membranes (Figure S5).

To further investigate the toxic effects of the Ag NPs, these were studied at varying concentrations (Figure. 4). Based on the EC50 values from the LDH assay, the Caco-2 monoculture was significantly more susceptible than the co-culture to cytotoxic damage by Ag NPs, while there was at least a slightly increased susceptibility of the inflamed compared to the non-inflamed co-culture (Figure 4A). It was also tested via LDH assay, if Ag ions released from the NPs were responsible for the toxic effect. There was no cytotoxicity in cells that were incubated with particle-free supernatants, which had been prepared by removal of intact Ag NPs following incubation (Figure S6).

Transepithelial electrical resistance was also measured before and $24 \mathrm{~h}$ after Ag NP incubation (Figure 4B). However, for Ag
NPs, the epithelial barrier was disrupted (as indicated by a steep drop of the TEER) as soon as the particle concentration reached a certain critical value $\left(156.25 \mu \mathrm{g} / \mathrm{cm}^{2}\right)$. Monoculture was most sensitive to Ag NPs followed by the inflamed and the non-inflamed co-culture. With the highest Ag concentration $\left(625 \mu \mathrm{g} / \mathrm{cm}^{2}\right)$, the TEER values showed an abrupt decrease similar to cells incubated with $1 \%$ Triton $\mathrm{X}$, which served as positive control (data not shown). After incubation with $\mathrm{Au}$ NPs, barrier properties were preserved even at a NP concentration of $80 \mu \mathrm{g} / \mathrm{cm}^{2}$ (Figure S7).

\section{Impact of NPs on inflammatory responses}

In order to identify possible sub-lethal effects on the epithelial cells, inflammatory responses elicited during cellular exposure to NPs were evaluated by measuring the secretion of cytokines. For this purpose, the apparently non-toxic $15 \mathrm{~nm} \mathrm{Au} \mathrm{NPs} \mathrm{were}$ chosen and compared with the evidently more toxic Ag NPs.

Co-cultures as well as Caco-2 monocultures were exposed to $\mathrm{Ag}$ and $\mathrm{Au}$ NPs for $24 \mathrm{~h}$. The inflammatory response was evaluated by monitoring the stimulation of IL- 8 release, both on protein (Figure 5A and B) and transcriptional level (Figure 5C and D). In Caco-2 monoculture, a slight increase in IL-8 release was observed only with the highest Ag NP concentration (Figure 5A). However, in the co-cultures, IL-8 release was observed already after incubation with the lowest tested Ag NP concentrations, which shows that these NPs induce inflammatory reactions even at concentrations, which are not yet toxic to the cells. Again, significant differences between the inflamed and non-inflamed state were observed. Surprisingly, the non-inflamed co-culture released more IL- 8 than the inflamed one when exposed to Ag NPs. The results with Ag NPs from real-time experiments agreed well with protein expression data (Figure $5 \mathrm{C}$ ).

Incubation of cell models with low concentrations of Au NPs did not induce a notable increase of IL- 8 production in the monoculture at any tested concentration (Figure 5B and D). The inflamed co-cultures naturally released more IL-8 than the healthy ones; however, IL-8 did not increase substantially with $\mathrm{Au} \mathrm{NP}$ dose in any of the models. The IL- 8 levels were approximately 10 times lower than those observed following $\mathrm{Ag}$ NP treatment.

\section{Discussion}

The application of nanotechnology in various areas is dramatically increasing. The high interest in ENs for medical application and their increased use in technological or consumable products have raised concerns about their impact on living systems. Several examples are addressed in the review by Arora et al. (2012), which emphasize the need to understand how nanomaterials interact with biological systems in order to avoid cytotoxic effects. By using appropriate in vitro models the safety of nanomaterials can be reliably evaluated. Therefore, the purpose of this study was to develop a 3D intestinal co-culture model composed of cell lines, which mimics healthy and diseased conditions and is suitable to assess NP-cell interactions.

Co-culture models have been widely accepted (RothenRutishauser et al., 2005, 2008) and used in several studies, e.g. to evaluate NP toxicity when exposed to the lungs by inhalation (Muller et al., 2010; Rothen-Rutishauser et al., 2008). Regarding the intestinal epithelium, i.e. after oral ingestion, most of the models focus on co-cultures to study absorption, particle celltranslocation or particle-mucus interaction (Schimpel et al., 2014). In our system, we focused on an intestinal model that could mimic either healthy or inflamed conditions. Caco-2 cells formed a confluent monolayer and functional tight junctions over three weeks. Despite being in the collagen laver, macrophages 

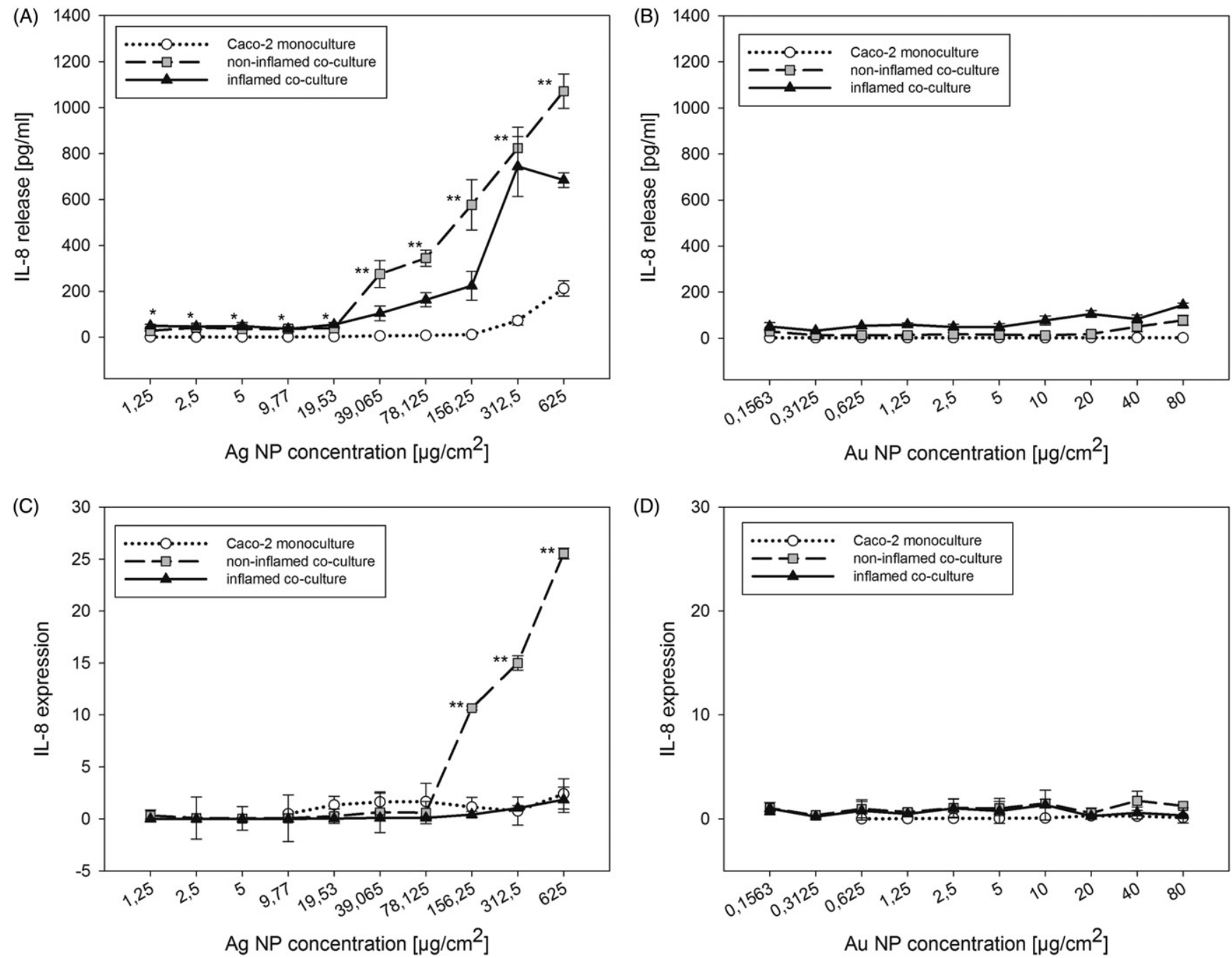

Figure 5. IL-8 production after incubation with Ag and Au NPs. Non-inflamed Caco-2, inflamed and non-inflamed co-culture were incubated with NPs for $24 \mathrm{~h}$. IL-8 protein release was measured by FACS (A, B) (after incubation with Ag NPs differences between co-cultures and monoculture were significant for every tested concentration). IL-8 expression was measured via real time PCR (C, D) and expression was calculated with housekeeping gene $\beta$-actin (mean $\pm \mathrm{SD}, n=6$ from two independent experiments, ${ }^{*} p<0.05$, ${ }^{*} p<0.001$, for protein measurement differences between co-cultures and monoculture were significant for every tested $\mathrm{Ag}$ and $\mathrm{Au}$ concentration).

could interact with Caco-2 cells, being located between them or on top of the epithelium. Interestingly, this interaction did not disrupt the intercellular barrier of the tight junctions as shown by uncompromised TEER value measurements. Other studies have shown that THP-1 cells co-cultivated with Caco- 2 cells induced epithelial cell death mainly due to TNF- $\alpha$ secretion by THP-1 cells (Satsu et al., 2006). However, this was not observed in our system.

After inflammation the 3D co-culture model showed lower TEER values. This effect was reversible when IL-1 $\beta$ was removed after two days, which is in agreement with the previous model based on primary cells (Leonard et al., 2010). Inflammation leads to a loss of the epithelial barrier function because tight junctions open, but without the occurrence of cell death (Bertiaux-Vandaele et al., 2011). This effect occurs because several tight junction protein as occludin or Claudin 5 and 8 are down-regulated due to the inflammation (Schulzke et al., 2009; Zeissig et al., 2007). Other groups reported significantly decreasing TEER values after treatment of Caco-2 cells with lipopolysaccharide (Guo et al., 2013), corroborating our data.

The inflammation resulted in an increase of IL-8 release that was higher in the co-culture compared to the epithelial monoculture. This is likely due to the presence of immune-competent cells, and the level of cytokine production was comparable between models containing cell lines or primary immune cells. Many experiments with nanomaterials in co-culture models have shown a clear difference to the results of monocultures (Lehmann et al., 2009, 2010; Muller et al., 2010). A co-culture model of epithelial-like transformed cells (HeLa) together with macrophage-like cells (U937) has been established to investigate the occurrence of inflammation after treatment with different causative agents of chronic periodontitis, emphasizing the important role of macrophages to model inflammatory conditions (Bodet et al., 2006). Immune-competent cells may influence epithelial barrier properties during inflammation as shown in studies using endothelial cells (Hermanns et al., 2010); however, in our model this was not observed.

To explore the possible difference in toxicity triggered by ENs, we studied the interactions of $\mathrm{Au}, \mathrm{TiO}_{2}$ and $\mathrm{Ag}$ NPs with the 3D co-culture and with Caco-2 monolayers. For Au NPs some studies have shown that they are not toxic; however, other studies have found a low toxicity of Au NPs (Kuhlbusch et al., 2011; Sung et al., 2011; Perreault et al., 2012). It is possible that the toxicity depends on the particle-size (Zhang et al., 2011). Here, two differently-sized Au NPs were used, 15 and $80 \mathrm{~nm}$, but no toxic effect was detected in any cell culture model. However, IL- 8 gene expression was slightly increased after exposure with the $15 \mathrm{~nm}$ $\mathrm{Au}$ NPs in the triple culture models. It has been also shown that 
$\mathrm{Au}$ NPs induce an inflammatory response in a co-culture model of the human airways (Rothen-Rutishauser et al., 2007). In vivo assays have also shown a significant expression of proinflammatory markers, such as IL-6, IL-1 $\beta$ (Cho et al., 2009) or $\mathrm{TNF} \alpha$ (Rothen-Rutishauser et al., 2007) after exposure to different $\mathrm{Au}$ NPs. Although Au NPs did not cause any toxic effects in our systems, they were able to induce inflammatory responses that were evident only in the co-culture, which agrees with other studies that compared co-cultures and monocultures of epithelial cells (H441) and endothelial cells (ISO-HAS-1) (Kasper et al., 2011). Clearly, cytokine measurement is a reliable endpoint in a toxicological assessment of nanomaterials, as inflammatory effects can occur at subtoxic concentrations. It has been shown that a co-culture system with epithelial and immune cells can show an amplified inflammatory response when in contact with particles (Alfaro-Moreno et al., 2008). In our model, this amplified effect can be explained by the presence of the macrophages and dendritic cells, which are both responsible for the cytokine release. When we evaluated the uptake of Au NPs by the cells in the $3 \mathrm{D}$ co-culture, we found that these particles were mostly internalized by Caco- 2 cells rather than by macrophages. However, in the case of Ag particles, increased cytokine production by immune cells may occur in response to cell death of Caco-2 cells and also possibly immune cells.

NM101 $\mathrm{TiO}_{2}$ nanoparticles did not induce toxic effects or inflammation in our models, which is a good sign since $\mathrm{TiO}_{2}$ NPs are often found in food or toothpaste (Weir et al., 2012) and can easily reach the human intestine. Nevertheless, other studies have shown a low toxicity of differently sized $\mathrm{TiO}_{2}$ nanoparticles to mouse macrophages (Zhang et al., 2012) or epithelial lung cells (Hsiao \& Huang, 2011). However, both studies used monoculture models, while a study with a co-culture model of the human airway wall with macrophages, dendritic cells and epithelial cells corroborates our results, as no toxicity or pro-inflammatory reaction was observed (Rothen-Rutishauser et al., 2007).

Ag NPs clearly demonstrated toxicity in all cell culture models used in the current work. LDH results showed that these particles induced a dose-dependent toxicity in Caco- 2 cells and co-cultures in healthy and inflamed conditions. However, EC50 values show that the Caco-2 monoculture is more sensitive to the toxic NPs than the co-culture models. As a clear interaction between immune-competent cells and Caco- 2 cells was observed in the 3D co-culture, it is believed that this interaction might help to reduce the sensitivity to the toxic NPs. The toxic effect of Ag NPs was also observed by the barrier disruption, in which TEER values decreased after the incubation with higher Ag NP concentrations. This considerable decrease in the TEER values correlated well with the increased cell death, as inferred from a high LDH release. Furthermore, the non-inflamed triple culture was less sensitive to the NPs than the inflamed one, in agreement with the data from the LDH assay; this suggests that the inflamed triple culture is already stressed from the IL- $1 \beta$ treatment. Our experiments show that IL-8 release is higher in the non-inflamed co-culture than in the already inflamed one after incubation with Ag NPs. This might be explained by the fact that the inflamed co-culture is already stressed by the inflammation. High release of IL- 8 was also observed in other studies that addressed the toxic potential of Ag NPs in human renal proximal tubule epithelial cells $(\mathrm{HK}-2)$ (Kermanizadeh et al., 2013b), C3A cells (Kermanizadeh et al., 2013a), and also in mice (Gaiser et al., 2012). It is not clear why the Ag NPs show such a high toxicity to the cells. Several studies suggest that released Ag ions are responsible for cytotoxic effects (Lee et al., 2012; Newton et al., 2013). Therefore, we did experiments with particle free supernatant from Ag NPs and could not detect toxic effects (Figure S5), which shows that released Ag ions are not the reason for the cytotoxicity.
The measurement of interleukins as readouts of NP interactions with cells allows the investigation of other cellular responses besides membrane permeability and metabolic activity, i.e. inflammation. Indeed interleukins are important biomarkers also for inflammation in vivo as shown, for example, in the high release of IL-8 in the GI of IBD patients (Muzes et al., 2012). Therefore, inflammatory response is an important parameter to be addressed in the interaction between NPs and living systems and should be included in safety testing of NPs and nanoparticulate pharmaceuticals. Even if some nanomaterials do not show any toxic effects on cell culture models, it is possible that they induce inflammatory reactions, which could be observed in our experiments with Au NPs. This is also supported by other studies that show an amplified inflammatory response when a co-culture of epithelial and immune-competent cells interact with particles (Alfaro-Moreno et al., 2008). Indeed, several experiments exploring nanomaterial interactions with co-culture and monoculture models showed a clear difference between such models (Lehmann et al., 2009, 2010; Muller et al., 2010), which supports the use of more complex in vitro systems to investigate in depths the interactions between NPs and biological systems.

\section{Conclusion}

The co-culture model established here represents a new approach to study intestinal inflammation under controlled conditions in vitro, an important issue for safety testing of nanomaterials. The replacement of primary immune cells by cell lines THP-1 and MUTZ-3 resulted in a more robust system, which is useful for higher throughput assays, while immunocompetence and epithelial cell differentiation remain preserved. Furthermore, this model has the advantage of being less time consuming and less variable than models based on primary cells obtained from blood samples of different patients. The differences between healthy and inflamed tissue in terms of barrier permeability support the importance of a model capable of mimicking diseased conditions, which results in different outcomes during the testing of new formulations. The immune-competent cells in this model play an important role in the scenario of NP-cell interactions, because they are responsible for the release of pro-inflammatory cytokines, an important endpoint to be considered in safety testing of nanomaterials. Thus, this study provides a new 3D co-culture model of the inflamed intestine suitable for the assessment of toxic and inflammatory effects of engineered nanomaterials as well as nanoparticulate pharmaceuticals in the intestine.

\section{Acknowledegments}

We thank the Electron Microscopy Unit for Biological Sciences, Department of Biosciences, University of Oslo. Thanks to Dr Wolfgang Kreyling from Helmholtz Zentrum, Munich, for providing the 15 and $80 \mathrm{~nm}$ Au nanoparticles. Thanks to Prof. Vicki Stone and Dr Birgit Gaiser from Heriot-Watt University, Edinburgh, for helpful discussions about FACS results. We also thank Dr. Sarah Gordon from Helmholtz Institute for Pharmaceutical Research, Saarbrücken, for proof-reading the manuscript and Petra König (Helmholtz Institute for Pharmaceutical Research) and Marijas Jurisic (Saarland University) for their help in cell culture and FACS experiments.

\section{Declaration of interest}

This work was funded from the EU FP7 project InLiveTox (NMP4-SL-2009-228625).

\section{References}

Alexis NE, Huang YC, Rappold AG, Kehrl H, Devlin R, Peden DB. 2014. Patients with asthma demonstrate airway inflammation after exposure 
to concentrated ambient particulate matter. Am J Respir Crit Care Med 190:235-7.

Alfaro-Moreno E, Nawrot TS, Vanaudenaerde BM, Hoylaerts MF, Vanoirbeek JA, Nemery B, Hoet PH. 2008. Co-cultures of multiple cell types mimic pulmonary cell communication in response to urban PM10. Eur Respir J 32:1184-94.

Arora S, Rajwade JM, Paknikar KM. 2012. Nanotoxicology and in vitro studies: the need of the hour. Toxicol Appl Pharmacol 258:151-65.

Ayala V, Herrera AP, Latorre-Esteves M, Torres-Lugo M, Rinaldi C. 2013. Effect of surface charge on the colloidal stability and in vitro uptake of carboxymethyl dextran-coated iron oxide nanoparticles. J Nanopart Res 15:1874.

Beer C, Foldbjerg R, Hayashi Y, Sutherland DS, Autrup H. 2012. Toxicity of silver nanoparticles - nanoparticle or silver ion? Toxicol Lett 208: 286-92.

Beloqui A, Coco R, Memvanga PB, Ucakar B, des Rieux A, Preat V. 2014. $\mathrm{pH}-$ Sensitive nanoparticles for colonic delivery of curcumin in inflammatory bowel disease. Int J Pharm 473:203-12.

Bertiaux-Vandaele N, Youmba SB, Belmonte L, Lecleire S, Antonietti M, Gourcerol G, et al. 2011. The expression and the cellular distribution of the tight junction proteins are altered in irritable bowel syndrome patients with differences according to the disease subtype. Am J Gastroenterol 106:2165-73.

Bisping G, Lugering N, Lutke-Brintrup S, Pauels HG, Schurmann G, Domschke W, Kucharzik T. 2001. Patients with inflammatory bowel disease (IBD) reveal increased induction capacity of intracellular interferon-gamma (IFN-gamma) in peripheral CD8+ lymphocytes cocultured with intestinal epithelial cells. Clin Exp Immunol 123:15-22.

Bodet C, Chandad F, Grenier D. 2006. Inflammatory responses of a macrophage/epithelial cell co-culture model to mono and mixed infections with Porphyromonas gingivalis, Treponema denticola, and Tannerella forsythia. Microbes Infect 8:27-35.

Cho WS, Kim S, Han BS, Son WC, Jeong J. 2009. Comparison of gene expression profiles in mice liver following intravenous injection of 4 and 100 nm-sized PEG-coated gold nanoparticles. Toxicol Lett 191: 96-102.

Desjardins M, Griffiths G. 2003. Phagocytosis: latex leads the way. Curr Opin Cell Biol 15:498-503.

Dobrovolskaia MA, Aggarwal P, Hall JB, McNeil SE. 2008. Preclinical studies to understand nanoparticle interaction with the immune system and its potential effects on nanoparticle biodistribution. Mol Pharm 5: 487-95.

Dobrovolskaia MA, McNeil SE. 2007. Immunological properties of engineered nanomaterials. Nat Nanotechnol 2:469-78.

Fang HW, Fang SB, Chiang Chiau JS, Yeung CY, Chan WT, Jiang CB, et al. 2010. Inhibitory effects of Lactobacillus casei subsp. rhamnosus on Salmonella lipopolysaccharide-induced inflammation and epithelial barrier dysfunction in a co-culture model using Caco-2/peripheral blood mononuclear cells. J Med Microbiol 59:573-9.

Fisichella M, Berenguer F, Steinmetz G, Auffan M, Rose J, Prat O. 2012. Intestinal toxicity evaluation of $\mathrm{TiO}_{2}$ degraded surface-treated nanoparticles: a combined physico-chemical and toxicogenomics approach in caco-2 cells. Part Fibre Toxicol 9:18.

Foger F, Kopf A, Loretz B, Albrecht K, Bernkop-Schnurch A. 2008. Correlation of in vitro and in vivo models for the oral absorption of peptide drugs. Amino Acids 35:233-41.

Frick A, Moller H, Wirbitzki E. 1998. Biopharmaceutical characterization of oral immediate release drug products. In vitro/in vivo comparison of phenoxymethylpenicillin potassium, glimepiride and levofloxacin. Eur J Pharm Biopharm 46:305-11.

Frohlich E, Roblegg E. 2012. Models for oral uptake of nanoparticles in consumer products. Toxicology 291:10-17.

Gaiser BK, Fernandes TF, Jepson M, Lead JR, Tyler CR, Stone V. 2009. Assessing exposure, uptake and toxicity of silver and cerium dioxide nanoparticles from contaminated environments. Environ Health 8:S2.

Gaiser BK, Fernandes TF, Jepson MA, Lead JR, Tyler CR, Baalousha M, et al. 2012. Interspecies comparisons on the uptake and toxicity of silver and cerium dioxide nanoparticles. Environ Toxicol Chem 31: 144-54.

Gaiser BK, Hirn S, Kermanizadeh A, Kanase N, Fytianos K, Wenk A, et al. 2013. Effects of silver nanoparticles on the liver and hepatocytes in vitro. Toxicol Sci 131:537-47.

Guo S, Al-Sadi R, Said HM, Ma TY. 2013. Lipopolysaccharide causes an increase in intestinal tight junction permeability in vitro and in vivo by inducing enterocyte membrane expression and localization of TLR-4 and CD14. Am J Pathol 182:375-87.
Hermanns MI, Kasper J, Dubruel P, Pohl C, Uboldi C, Vermeersch V, et al. 2010. An impaired alveolar-capillary barrier in vitro: effect of proinflammatory cytokines and consequences on nanocarrier interaction. J R Soc Interface 7:S41-54.

Hsiao IL, Huang YJ. 2011. Effects of various physicochemical characteristics on the toxicities of $\mathrm{ZnO}$ and $\mathrm{TiO}$ nanoparticles toward human lung epithelial cells. Sci Total Environ 409:1219-28.

Kasper J, Hermanns MI, Bantz C, Maskos M, Stauber R, Pohl C, Unger RE, Kirkpatrick JC. 2011. Inflammatory and cytotoxic responses of an alveolar-capillary coculture model to silica nanoparticles: comparison with conventional monocultures. Part Fibre Toxicol 8:6.

Kermanizadeh A, Pojana G, Gaiser BK, Birkedal R, Bilanicova D, Wallin $\mathrm{H}$, et al. 2013a. In vitro assessment of engineered nanomaterials using a hepatocyte cell line: cytotoxicity, pro-inflammatory cytokines and functional markers. Nanotoxicology 7:301-13.

Kermanizadeh A, Vranic S, Boland S, Moreau K, Baeza-Squiban A, Gaiser BK, Andrzejczuk LA, Stone V. 2013b. An in vitro assessment of panel of engineered nanomaterials using a human renal cell line: cytotoxicity, pro-inflammatory response, oxidative stress and genotoxicity. BMC Nephrol 14:96.

Kish L, Hotte N, Kaplan GG, Vincent R, Tso R, Ganzle M, et al. 2013. Environmental particulate matter induces murine intestinal inflammatory responses and alters the gut microbiome. PLoS One 8:e62220.

Koeneman BA, Zhang Y, Westerhoff P, Chen Y, Crittenden JC, Capco DG. 2010. Toxicity and cellular responses of intestinal cells exposed to titanium dioxide. Cell Biol Toxicol 26:225-38.

Kuhlbusch TA, Asbach C, Fissan H, Gohler D, Stintz M. 2011. Nanoparticle exposure at nanotechnology workplaces: a review. Part Fibre Toxicol 8:22.

Lee YJ, Kim J, Oh J, Bae S, Lee S, Hong IS, Kim SH. 2012. Ion-release kinetics and ecotoxicity effects of silver nanoparticles. Environ Toxicol Chem 31:155-9.

Lefebvre DE, Venema K, Gombau L, Valerio Jr LG, Raju J, Bondy GS, et al. 2014. Utility of models of the gastrointestinal tract for assessment of the digestion and absorption of engineered nanomaterials released from food matrices. Nanotoxicology [Epub ahead of print]. doi:10.3109/17435390.2014.948091.

Lehmann AD, Blank F, Baum O, Gehr P, Rothen-Rutishauser BM. 2009. Diesel exhaust particles modulate the tight junction protein occludin in lung cells in vitro. Part Fibre Toxicol 6:26.

Lehmann AD, Parak WJ, Zhang F, Ali Z, Rocker C, Nienhaus GU, et al. 2010. Fluorescent-magnetic hybrid nanoparticles induce a dosedependent increase in proinflammatory response in lung cells in vitro correlated with intracellular localization. Small 6:753-62.

Leonard F, Ali H, Collnot EM, Crielaard BJ, Lammers T, Storm G, Lehr CM. 2012. Screening of budesonide nanoformulations for treatment of inflammatory bowel disease in an inflamed 3D cell-culture model. ALTEX 29:275-85.

Leonard F, Collnot EM, Lehr CM. 2010. A three-dimensional coculture of enterocytes, monocytes and dendritic cells to model inflamed intestinal mucosa in vitro. Mol Pharm 7:2103-19.

Loeschner K, Hadrup N, Qvortrup K, Larsen A, Gao X, Vogel U, et al. 2011. Distribution of silver in rats following 28 days of repeated ora exposure to silver nanoparticles or silver acetate. Part Fibre Toxicol $\underline{8: 18 .}$

Mahmud N, Weir DG. 2001. The urban diet and Crohn's disease: is there a relationship?' Eur J Gastroenterol Hepatol 13:93-5.

Mironava T, Hadjiargyrou M, Simon M, Jurukovski V, Rafailovich MH. 2010. Gold nanoparticles cellular toxicity and recovery: effect of size, concentration and exposure time. Nanotoxicology 4:120-37.

Muller L, Riediker M, Wick P, Mohr M, Gehr P, Rothen-Rutishauser B. 2010. Oxidative stress and inflammation response after nanoparticle exposure: differences between human lung cell monocultures and an advanced three-dimensional model of the human epithelial airways. J R Soc Interface 7:S27-40.

Muzes G, Molnar B, Tulassay Z, Sipos F. 2012. Changes of the cytokine profile in inflammatory bowel diseases. World J Gastroenterol 18: 5848-61.

Newton KM, Puppala HL, Kitchens CL, Colvin VL, Klaine SJ. 2013. Silver nanoparticle toxicity to Daphnia magna is a function of dissolved silver concentration. Environ Toxicol Chem 32:2356-64.

Perreault F, Melegari SP, Fuzinatto CF, Bogdan N, Morin M, Popovic R, Matias WG. 2012. Toxicity of PAMAM-coated gold 
nanoparticles in different unicellular models. Environ Toxicol 29: 328-36.

Powell JJ, Faria N, Thomas-McKay E, Pele LC. 2010. Origin and fate of dietary nanoparticles and microparticles in the gastrointestinal tract. J Autoimmun 34:J226-33.

Rothen-Rutishauser B, Mueller L, Blank F, Brandenberger C, Muehlfeld C, Gehr P. 2008. A newly developed in vitro model of the human epithelial airway barrier to study the toxic potential of nanoparticles. ALTEX 25:191-6.

Rothen-Rutishauser B, Muhlfeld C, Blank F, Musso C, Geh P. 2007. Translocation of particles and inflammatory responses after exposure to fine particles and nanoparticles in an epithelial airway model. Part Fibre Toxicol 4:9.

Rothen-Rutishauser BM, Kiama SG, Gehr P. 2005. A three-dimensional cellular model of the human respiratory tract to study the interaction with particles. Am J Respir Cell Mol Biol 32:281-9.

Satsu H, Ishimoto Y, Nakano T, Mochizuki T, Iwanaga T, Shimizu M. 2006. Induction by activated macrophage-like THP-1 cells of apoptotic and necrotic cell death in intestinal epithelial Caco-2 monolayers via tumor necrosis factor-alpha. Exp Cell Res 312:3909-19.

Schimpel C, Teubl B, Absenger M, Meindl C, Frohlich E, Leitinger G, et al. 2014. Development of an advanced intestinal in vitro triple culture permeability model to study transport of nanoparticles. Mol Pharm 11:808-18.

Schulzke JD, Ploeger S, Amasheh M, Fromm A, Zeissig S, Troeger H, et al. 2009. Epithelial tight junctions in intestinal inflammation. Ann N Y Acad Sci 1165:294-300.
Sung JH, Ji JH, Park JD, Song MY, Song KS, Ryu HR, et al. 2011. Subchronic inhalation toxicity of gold nanoparticles. Part Fibre Toxicol 8:6.

Theegarten D, Boukercha S, Philippou S, Anhenn O. 2010. Submesothelial deposition of carbon nanoparticles after toner exposition: case report. Diagn Pathol 5:77.

Ucciferri N, Collnot EM, Gaiser BK, Tirella A, Stone V, Domenici C, et al. 2014. In vitro toxicological screening of nanoparticles on primary human endothelial cells and the role of flow in modulating cell response. Nanotoxicology 8:697-708.

Walter E, Janich S, Roessler BJ, Hilfinger JM, Amidon GL. 1996. HT29MTX/Caco-2 cocultures as an in vitro model for the intestinal epithelium: in vitro-in vivo correlation with permeability data from rats and humans. J Pharm Sci 85:1070-6.

Weir A, Westerhoff P, Fabricius L, Hristovski K, von Goetz N. 2012. Titanium dioxide nanoparticles in food and personal care products. Environ Sci Technol 46:2242-50.

Zeissig S, Burgel N, Gunzel D, Richter J, Mankertz J, Wahnschaffe U, et al. 2007. Changes in expression and distribution of claudin 2, 5 and 8 lead to discontinuous tight junctions and barrier dysfunction in active Crohn's disease. Gut 56:61-72.

Zhang J, Song W, Guo J, Sun Z, Li L, Ding F, Gao M. 2012. Cytotoxicity of different sized $\mathrm{TiO}_{2}$ nanoparticles in mouse macrophages. Toxicol Ind Health 29:523-33.

Zhang XD, Wu D, Shen X, Liu PX, Yang N, Zhao B, et al. 2011. Sizedependent in vivo toxicity of PEG-coated gold nanoparticles. Int J Nanomedicine 6:2071-81.

Supplementary material available online

Supplementary Tables S1, S2

Figures S1-S7. 